\title{
PRESERVACIÓN AUTOMÁTICA DE DISCONTINUIDADES DE SUPERFICIE BASADA EN LA ESTIMACIÓN DE LA TORSIÓN DE CURVAS DE CONTORNO PARA LA RECONSTRUCCIÓN DE OBJETOS TRIDIMENSIONALES
}

\section{AUTOMATIC PRESERVATION OF SURFACE DISCONTINUITIES BASED ON TORSION ESTIMATION OF THE CONTOUR CURVES FOR THE RECONSTRUCTION OF THREE- DIMENSIONAL OBJECTS}

\author{
Germán Sánchez Torres ${ }^{1} \quad$ John William Branch ${ }^{2}$ \\ Recibido 8 de abril de 2008, aceptado 30 de marzo de 2009 \\ Received: April 8, 2008 Accepted: March 30, 2009
}

\begin{abstract}
RESUMEN
Los diferentes métodos de ajuste de superficies desde datos dispersos asumen que la superficie reconstruida no presenta ningún tipo de discontinuidad. Las discontinuidades en las superficies son obtenidas a partir de la forma del objeto original y estas aparecen principalmente en el proceso de adquisición debido a problemas de oclusión. Tradicionalmente, para la representación de superficies que tienen discontinuidades se realiza un proceso manual adicional después de la etapa final de ajuste. En este artículo se propone un método para detectar y preservar discontinuidades automáticamente en superficies de topología arbitraria, así como un método para la reducción de los puntos a interpolar, que permite disminuir el costo computacional asociado a la solución del interpolante. Inicialmente, el método realiza una clasificación basada en la estimación de la varianza de la torsión de la curva del contorno. Cuando el valor de la varianza en el contorno de los huecos es muy bajo, entonces se considera que este hueco debe ser preservado. Luego, la superficie descrita por el conjunto de puntos seleccionados es interpolada usando Funciones de Base Radial. Por último, la superficie generada es cortada con cada contorno identificado como preservable.
\end{abstract}

Palabras clave: Reconstrucción de superficies, funciones de base radial, datos de rango, estrategia evolutiva.

\section{ABSTRACT}

Different methods for fitting surfaces from dispersed data interpolation assume the reconstructed surface does not have any type of discontinuity. Discontinuities in the surfaces are obtained from the original shape of the object and they appear mainly in the acquisition process due to occlusion problems. For digital representation of surfaces with discontinuities is commonly found an additional manual process, which is applied after the final stage of fitting surface. In this paper, two methods are proposed, one of them with the aim of detect and preserve discontinuities in surfaces with arbitrary topology, and the other one to reduce the amount of points to be interpolated, decreasing the computational cost. Initially, the method carries out a classification based on estimation of the torsion variance of the contour curve. When the holes contour has very low values of variances, it is considered that this hole must be preserved. Then, the surface described by the selected set of points is interpolated using Radial Basis Functions. Finally, the generated surface is trimmed with each contour identified like preservable.

Keywords: Surface reconstruction, radial basis functions, range data, evolutionary strategy.

\section{INTRODUCCIÓN}

La reconstrucción de superficies es el proceso mediante el cual objetos reales son reproducidos digitalmente, manteniendo sus características físicas como la dimensión, el volumen y la forma. La generación de estos modelos tiene diversos campos de aplicación, tales como el reconocimiento de objetos, reconocimiento facial para la identificación, el modelado de piezas arqueológicas, entre otros. Un modelo digital es creado a partir de información

\footnotetext{
1 Universidad del Magdalena. Facultad de Ingeniería. Programa de Sistemas. Santa Marta, Colombia. E-mail: gsanchez@unimagdalena.edu.co

2 Universidad Nacional de Colombia. Escuela de Sistemas e Informática. Medellín, Colombia. E-mail: jwbranch@unalmed.edu.co
} 
geométrica adquirida directamente del objeto real y almacenado en imágenes de rango. Las imágenes de rango constituyen una manera no invasiva y de alta precisión para obtener información acerca de la geometría de un objeto, tomando distancias medidas desde una posición conocida de un sensor hasta puntos sobre la superficie del objeto. El posterior tratamiento de la información adquirida consiste en un conjunto de etapas que permiten finalmente obtener la representación digital del objeto, a saber, adquisición, registro, integración y ajuste.

La base del problema de ajuste de superficies mediante interpolación consiste en encontrar una superficie que interpole un número finito de $N$ puntos $\mathrm{P}=\left\{\mathrm{x}_{1}, \mathrm{x}_{2}, \ldots, \mathrm{x}_{\mathrm{n}-1}\right.$, $\mathrm{x}_{\mathrm{n}}$ \} en $\mathrm{R}^{3}$. Existen diferentes variantes del problema, el más simple de los casos ocurre cuando los datos están preescritos en una superficie en $\mathrm{R}^{2}$; en estos casos se mantiene una función bivariada $F(x, y)$, la cual toma ciertos valores para cualquier pareja variante $\left(x_{i}, y_{i}\right)$, esto es, $F\left(x_{i}, y_{i}\right)=z_{i}$ para todo $i=1, \ldots ., N$. Este problema es conocido como el problema de encontrar una función de reconstrucción. El caso más general de la interpolación de datos dispersos es conocido como el problema de superficie a superficie, en el cual los datos están preescritos en alguna superficie en $\mathrm{R}^{3}$; en este caso el problema es encontrar una superficie $S$ que interpole un conjunto finito de datos y otra superficie $W$ que interpole unos valores de función $w_{i j}$ estimados en cada punto de la superficie $\left(x_{i}, y_{i}, z_{i}\right) \in S$. Este problema puede ser visto como el problema de interpolar datos dispersos $\left(\mathrm{x}_{\mathrm{i}}, \mathrm{y}_{\mathrm{i}}, \mathrm{z}_{\mathrm{i}}, \mathrm{w}_{\mathrm{i}}\right)$ en $\mathrm{R}^{4}$, donde $\left(x_{i}, y_{i}, z_{i}\right)$ tiene la restricción de estar sobre la superficie en $R^{3}$. Los casos más populares son aquellos en donde $\mathrm{w}_{\mathrm{ij}}$ es un escalar $\mathrm{y}$ es generalmente una medida de distancia del punto a la superficie. La literatura en interpolación de datos dispersos es vasta; trabajos como el de Powell [20], Arge [1] y Buhmann [7, 8] describen métodos de interpolación con RBF (Radial Basis Functions). Las técnicas basadas en RBF están tomando importancia debido a que resultan ser muy atractivas para una gran cantidad de aplicaciones debido a la capacidad de reproducir forma de alta calidad, aun en presencia de datos irregularmente muestreados y con altos niveles de ruido.

Sin embargo, los métodos tradicionales de reconstrucción de superficies propuestos en la literatura asumen que la superficie completa es continua y no presenta discontinuidades, así cualquier irregularidad en los datos es interpretada como anomalía topológica y reparada para generar modelos digitales continuos. Esta idea es válida para un dominio reducido de objetos. Sin embargo, no todas las superficies de los objetos reales presentan estas características. Existe una variedad de objetos que presentan bordes que limitan su topología externa y bordes que denotan discontinuidades al interior de la superficie.
Este conjunto de discontinuidades deben ser preservadas en la representación digital final del objeto; parte de este trabajo está orientado a la clasificación automática de estas anomalías con el fin de mantenerlas en la representación final. De igual manera, este trabajo presenta una técnica para la construcción del interpolante, realizando una selección de un subconjunto de puntos, denominados centros de interpolación, con el objeto de disminuir el costo computacional asociado a la resolución del sistema de ecuaciones que se genera. Finalmente, estas dos etapas propuestas se apoyan en un mecanismo de recorte de superficies para generar superficies interpoladas que preserven discontinuidades del objeto real.

Este trabajo está organizado de la siguiente manera: en la sección Trabajos previos se hace una descripción de los trabajos realizados en el campo de la reconstrucción de superficies, mostrando diferentes enfoques y evidenciando que la preservación de discontinuidades es un procedimiento manual. En la sección Identificación de las discontinuidades de superficie se describe el procedimiento para la identificación automática de las discontinuidades de superficie de acuerdo a una representación triangular. En la sección Clasificación automática de discontinuidades se presenta el método propuesto para la clasificación de las discontinuidades como preservables (falsa anomalía) o no preservables (anomalía real). En la sección Generación de la superficie se describe el procedimiento general utilizado para la parametrización del interpolante utilizado en la etapa de ajuste y recorte automático de la superficie y en la sección final se presentan las Conclusiones y trabajos futuros.

\section{TRABAJOS PREVIOS}

Barrow y Tanenbaum [2] y Blicher [5] son los primeros en introducir el problema de la reconstrucción de superficies en el área de la visión por computador. Ellos implementaron una solución basada en la interpolación de superficies curvadas uniformemente. La solución aplica un algoritmo que se desarrolla con el uso de un arreglo de procesos paralelos simples que realizan variaciones locales iterativamente. Esta técnica es muy simple y presenta varios problemas, como, por ejemplo, el problema de las discontinuidades de la superficie.

Diferentes técnicas de interpolación pueden encontrarse en $[6,12,15,17]$.

Trabajos más recientes constituyen los acercamientos más completos e importantes al problema del ajuste de superficies; entre ellos existe una variedad muy amplia de técnicas utilizadas incluyendo también combinaciones 
de éstas. Los acercamientos mediante mallas triangulares y funciones splines han sido tema de una amplia gama de trabajos realizados en el área recientemente. Esta tendencia es en gran medida incentivada debido a que soluciones como las basadas en interpolación suelen ser muy costosas computacionalmente. Estas limitaciones actualmente están siendo mitigadas con el desarrollo de las capacidades de cómputo de las arquitecturas modernas, el aumento significativo en la capacidad de los dispositivos de almacenamiento y el desarrollo de técnicas más rápidas para la solución de densos sistemas de ecuaciones.

La interpolación mediante RBF desde el punto de vista teórico ha sido ampliamente utilizada en diferentes trabajos: Baxter [3] en su trabajo "The Interpolation Theory of Radial Basis Functions" investigó la sensibilidad de la interpolación de las RBF a los cambios en los valores de la función, para estudiar las matrices generadas por un conjunto de puntos dispuestos en grid regulares; adicionalmente, se analizaron los resultados de la interpolación de una amplia gama de familias de RBF. Otros trabajos relacionados son $[9,10,13,23]$.

Los trabajos teóricos y de aplicación realizados en la última década confirman una problemática relacionada con la interpolación de datos para reconstruir superficies, debido a que la teoría de la interpolación con RBF está bien definida y sustentada. El problema que emerge es el costo computacional anexo a este tipo de procedimientos comparados con otras técnicas de interpolación de datos como los splines que han sido ampliamente estudiadas en los últimos años.

En el 2005, Beatson [4] construyó un método para la rápida evaluación de interpolantes con funciones de base radial. El algoritmo propuesto está basado en una propiedad fundamental de funciones definidas condicionalmente negativas (o positivas); este método mostró un alto rendimiento sobre arquitecturas de clúster de estaciones de trabajo.

Otros trabajos proponen una técnica de ajuste adaptativo mediante RBF, como en Ohtake [19], el cual propone un método de selección de parámetros mediante el cual se garantiza la suavidad del modelo final; aquí el número de centros seleccionados para evaluar la función de base radial tiene influencia sobre la calidad del error.

Kojekine [16] utiliza la evaluación de una función de base radial de soporte compacto; este tipo de funciones necesita una menor complejidad computacional que otros modelos de RBF, ya que la utilización de estructuras de datos como los árboles Octales ayuda a reducir la complejidad de la búsqueda y clasificación de centros sobre los cuales una RBF es evaluada.

En este artículo, la tarea de ajustar superficies es realizada mediante técnicas de interpolación utilizando RBF de soporte global. El objetivo de la reconstrucción no es simplemente el de obtener un modelo digital de la superficie asumiendo que el objeto es completamente cerrado y que no posee discontinuidades; por el contrario, este trabajo presenta un procedimiento general de reconstrucción en el cual se asume que la superficie representada por el conjunto de puntos presenta discontinuidades obtenidas del objeto real, por lo que se deben preservar en la representación final del objeto. Los temas relacionados con el control de la suavidad no son tratados en profundidad, sin embargo, es conocido que las RBF permiten la configuración en el nivel de la precisión de la representación final [24].

Recientemente, una de las principales características deseables en los métodos de reconstrucción de superficies es la habilidad para llenar huecos o completar regiones faltantes. El proceso parte de un conjunto de imágenes que no cubren completamente el objeto, lo que ocasiona que la superficie generada esté incompleta. La falta de información es generada principalmente por propiedades reflectivas del material, o problemas de oclusión. Es decir, regiones inaccesibles para el sensor, lo que genera la necesidad de proponer procedimientos que permitan inferir la información de las regiones faltantes, ajustándose a las características geométricas del entorno de la ausencia en el objeto [32]. Los métodos de reconstrucción clásicos necesitan realizar el llenado de huecos mediante técnicas manuales de postprocesamiento aplicadas después de la etapa final de la reconstrucción de superficies, esto principalmente por la dificultad en diferenciar la naturaleza de la discontinuidad, esto es, si pertenece o no a la superficie real. En general, los métodos realizan la corrección de manera implícita durante la etapa de ajuste de superficies realizando una reconstrucción global del objeto [10, 32]; este enfoque tiene dos desventajas, primero, no permite reproducir huecos falsos, es decir, aquellos que pertenecen al objeto. Segundo, la calidad de la porción generada depende de la técnica utilizada y no del análisis de la geometría intrínseca en el objeto.

\section{IDENTIFICACIÓN DE LAS DISCONTINUIDADES DE SUPERFICIE}

Con el fin de identificar las discontinuidades al interior de la superficie se necesita inicialmente una representación triangular de la superficie creada a partir de la nube de puntos inicialmente censada. Diferentes trabajos describen 
diversas técnicas para la identificación de discontinuidades sobre mallas triangulares [25-27]. El procedimiento más sencillo y funcional con gran facilidad de implementación lo constituye el trabajo que se describe en [26]. Aquí, una discontinuidad en la superficie es fácilmente definida como una sucesión de aristas límite. Una arista límite se define como un borde de un triángulo que no es compartido por ningún otro triángulo. Es posible obtener sucesiones de aristas límite de dos tipos; las internas, que generalmente delimitan una frontera interior, este tipo de borde es en el que está basado este trabajo debido a que la mayoría de discontinuidades de superficie son de este tipo. Las externas, a su vez, delimitan o bien una "isla" dentro de una discontinuidad o bien definen los límites de una superficie como se muestra en la figura 1. Este trabajo está orientado a la detección de discontinuidades internas.

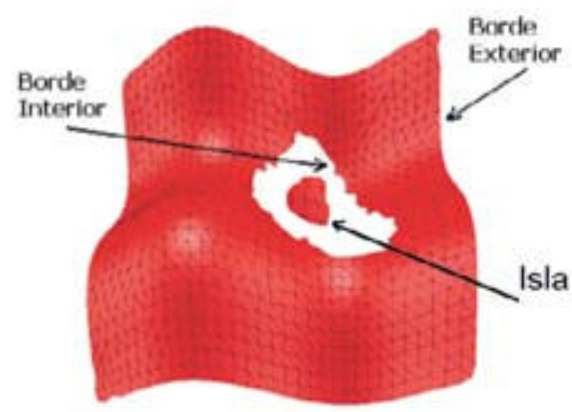

Figura 1. Diferentes tipos de bordes presentes en las superficies.

Inicialmente, el algoritmo toma una semilla triángulo ubicado en cualquier parte de la malla triangular, y realiza la búsqueda en toda la malla hasta que se encuentra un triángulo límite, a partir del cual se inicia una búsqueda recursiva para encontrar un camino cerrado de triángulos límite adyacentes. Esta búsqueda se realiza mediante la determinación de que por lo menos uno de los tres bordes o arista de un triángulo límite es una arista límite, y se realiza posteriormente una búsqueda de aristas límite adyacentes a cada triángulo que comparte algunos de esos vértices. El algoritmo es iterativo y termina el ciclo hasta que se encuentra nuevamente el triángulo inicial, a partir de aquí se considera que se ha encontrado un camino cerrado que denota una discontinuidad. Cada triángulo y arista límite son marcados para no repetirlos nuevamente en posteriores búsquedas. Estos pasos se realizan para cada triángulo límite que no ha sido marcado, hasta que no existan triángulos límite no marcados, este procedimiento puede verse en el Algoritmo 1.

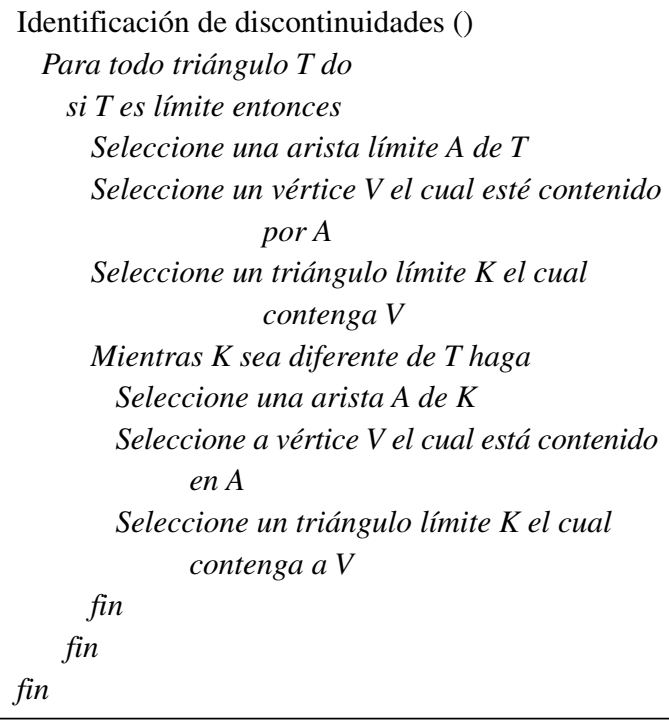

Algoritmo 1: Procedimiento para la identificación de discontinuidades propuesto.

\section{CLASIFICACIÓN AUTOMÁTICA DE DISCONTINUIDADES}

La preservación de bordes es importante debido a que pueden ser características que se presentan en los objetos reales. Los bordes externos representan generalmente los límites de la superficie y describen la forma externa general de la misma, por el contrario, los bordes internos describen las discontinuidades presentes en los objetos, que pueden o no ser del objeto real del cual se desea hallar la representación digital. Un acercamiento inicial hacia la automatización de este procedimiento consiste en analizar en el espacio el comportamiento de la curva que describe el contorno de la discontinuidad. Dos medidas desde la geometría estimada sobre la curva pueden ser utilizadas para este propósito, a saber, la curvatura y la torsión [31].

Así el objetivo de esta etapa es indicar si un hueco debe preservarse o no, tratando de determinar si la discontinuidad está presente en la superficie del objeto real o fue causada en alguna de las etapas intermedias de la reconstrucción (ver figura 2). Existen numerosas configuraciones posibles de discontinuidades en los objetos que hacen muy difícil establecer la pertenencia real de la discontinuidad a la superficie, es por esta razón que tradicionalmente la identificación de discontinuidades exige una interacción manual. Sin embargo, un intento por automatizar este procedimiento consiste en analizar la curva de contorno generada por cada uno de los huecos. El análisis de cada discontinuidad en este trabajo consiste en el estudio de la torsión de la curva del contorno de cada uno de estos. 
Este análisis se basa en la hipótesis de que cada hueco que pertenece a la superficie es de contorno suave y regular, pero los huecos generados por la oclusión tienden a presentar grandes irregularidades reflejadas en altas variaciones en su contorno y, por lo tanto, en la estimación de las torsiones en el contorno.

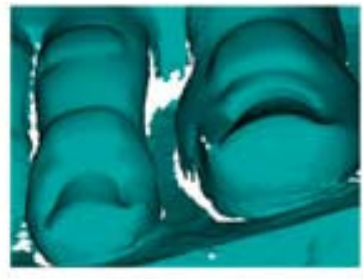

(a) Huecos generados por oclusión.

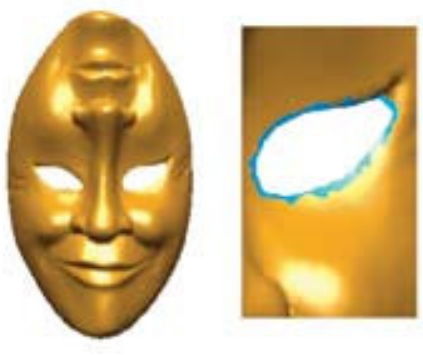

(b) Huecos generados por discontinuidad presente en el objeto real.

Figura 2. Diferentes tipos de huecos.

Debido a que la línea de contorno es una curva, esta puede ser estudiada y clasificada de acuerdo a sus propiedades geométricas como la curvatura y torsión. Para la clasificación de las curvas de contorno en este trabajo sólo se considera importante la torsión y no la curvatura. Entre otras razones, debido al problema de la no normalización en la escala de la medida de la curvatura y el hecho de que los huecos pueden tener cualquier nivel de curvatura dificulta el uso de esta como una técnica para la detección automática de huecos reales.

Geométricamente, la medida de la curvatura indica la velocidad de cambio de la curva sobre un mismo plano, por el contrario, la medida de la torsión indica el cambio de planos que realiza la curva en el espacio, es decir, indica el movimiento en el espacio de la curva. En discontinuidades de superficie que pertenecen a la superficie la curva de contorno no varía espacialmente mucho, debido a que los únicos cambios de esta estarán causados por la medida del error del dispositivo de adquisición, de otra forma los puntos serán muestreados sobre la misma superficie, lo que sugiere una suavidad en el cambio de la curva en el espacio entre los segmentos continuos de la curva del contorno. Las anomalías causadas por oclusión tienden a tener grandes variaciones debido a que los segmentos de región que no se pueden censar hacen que la curva de contorno cambie bruscamente en el espacio.

El estudio de la torsión de una curva depende del comportamiento del plano osculador. El plano osculador es el plano más cercano a la curva en un punto arbitrario $A$. Este plano pasa por el punto $A$ y contiene a la tangente $T$ y la normal $N$ de la curva en $A$. De un punto a otro a lo largo de una curva, la posición del plano osculador varía en forma similar a como lo hace la dirección de la tangente y permite caracterizar la curvatura. De forma análoga como con la curvatura, la variación del plano osculador es medida de acuerdo a la longitud de arco, esto es, si $\Psi$ es el ángulo entre los planos osculadores en un punto fijo A y un punto próximo $\mathrm{X}, \mathrm{y}$ si $\Delta s$ es la longitud de arco AX, entonces la torsión $\tau$ en el punto A se define como el límite de la razón entre el ángulo y la longitud de arco cuando esta tiende a infinito (ec. 1).

$$
\tau=\lim _{\Delta s \rightarrow 0} \frac{\Psi}{\Delta S}
$$

El signo de la torsión depende del lado de la curva hacia la que gira el plano osculador al moverse a lo largo de la curva. Sin embargo, desde la geometría diferencial las propiedades de una curva en un punto son aquellas propiedades que dependen de un entorno arbitrariamente pequeño, así las propiedades de este tipo se definen en términos de derivadas en el punto dado de la ecuación que define la curva (ec. 2). La estimación de la torsión se define como sigue:

$$
\tau=\frac{\left|\left(\vec{f}^{\prime} \times \vec{f}^{\prime \prime}\right) \cdot \vec{f}^{\prime \prime \prime}\right|}{\left|\left(\vec{f}^{\prime} \times \vec{f}^{\prime \prime}\right)\right|^{3}}
$$

Para estimar la torsión de la curva de contorno se necesita una ecuación $f(x)$ que la describa, debido a que se tiene un camino continuo y cerrado de aristas. Para obtener una ecuación del conjunto de aristas, estas se aproximan mediante curvas de Bézier, teniendo en cuenta que la función $f(x)$ que describa la curva debe ser diferenciable, hasta por lo menos en tercer grado. Una curva paramétrica de Bézier se define así (ver ecuaciones 3, 4 y 5):

$$
\begin{aligned}
& x(t)=\sum_{i=0}^{n} x_{i} B_{i, n}(t) \\
& y(t)=\sum_{i=0}^{n} y_{i} B_{i, n}(t)
\end{aligned}
$$




$$
z(t)=\sum_{i=0}^{n} z_{i} B_{i, n}(t)
$$

En general

$$
C b(t)=\sum_{i=0}^{n} p_{i} B_{i, n}(t)
$$

donde B son los polinomios de Berstein de tercer grado y se define así (ec. 7):

$$
\begin{aligned}
B_{0,3} & =(1-x)^{3} \\
B_{1,3} & =3 x(1-x)^{2} \\
B_{2,3} & =3 x^{2}(1-x) B_{3,3}=x^{3}
\end{aligned}
$$

El contorno es aproximado parcialmente mediante curvas de Bézier de grado tres, obtenido con conjuntos de cuatro puntos continuos hasta obtener la estimación de la torsión en todos los puntos que conforman el contorno. Una vez obtenidas las ecuaciones de los segmentos de la curva, la torsión es evaluada en el último punto; esto debido a que Bézier garantiza que la curva obtenida posea los puntos extremos del conjunto sobre el cual se calcula, de tal forma que el error de aproximación que se presenta en los puntos intermedios no afecta considerablemente a la estimación de la torsión (ver figura 3).

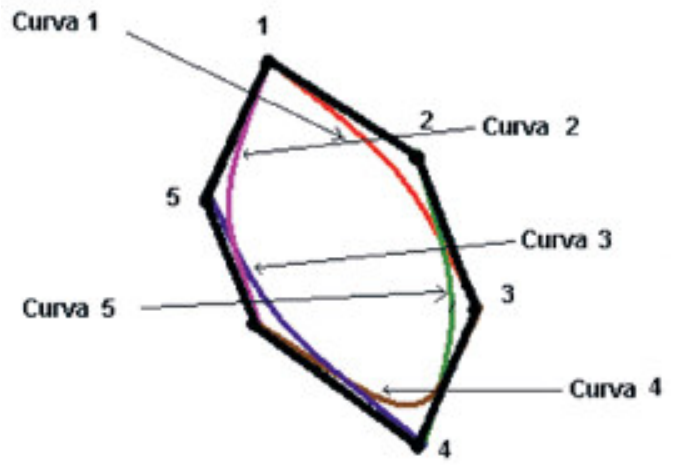

Figura 3. Aproximación de la curva de contorno mediante curvas de Bézier y puntos sobre los cuales se estima la torsión.

Finalmente se calcula la varianza de las torsiones para medir el nivel de dispersión de los valores medidos en cada punto; los huecos cuyos contornos posean una varianza de torsión superior a un umbral establecido se llenarán y se consideran no preservables, así:

$$
S_{\text {torsión }}=\frac{\sum\left(\tau_{i}-\bar{\tau}\right)^{2}}{n}
$$

La clasificación de preservable o no se determina comparando el valor estimado de la varianza frente al de un umbral U, así:

Para (Cada camino i de discontinuidad) haga

Si $($ Storsion $>U)$

Marcar como i como preservable

Sino

Descartar $i$

fpara

\section{GENERACIÓN DE LA SUPERFICIE}

Finalmente, con la clasificación de las discontinuidades el siguiente paso es generar una aproximación de la superficie. Esta aproximación será posteriormente recortada mediante la intersección de las curvas almacenadas y previamente marcadas como preservables. La aproximación de la superficie es realizada en este trabajo mediante la interpolación de un subconjunto de puntos de la nube inicial. Este subconjunto es seleccionado teniendo en cuenta afectar lo menos posible la precisión de la representación final y teniendo en cuenta que para que posteriormente se pueda recortar la superficie generada los puntos del contorno deben satisfacer la función de interpolación, es decir, deben hacer parte del subconjunto de puntos con el cual se construye el interpolante; a este subconjunto de puntos se llamará en adelante centros de la interpolación y estarán formados por tres sub-conjuntos seleccionados de acuerdo a las siguientes características:

1. Seleccionar un conjunto de puntos cuyas características geométricas sean representativas de su entorno.

2. Seleccionar todos los puntos que hagan parte de los contornos de los huecos clasificados como preservables.

3. Todos los puntos que correspondan a contornos externos, si los hay.

El objetivo de la característica 1 es disminuir el efecto de la pérdida de precisión con la reducción de puntos, así el objetivo es seleccionar puntos representativos por regiones, lo que sugiere algún tipo de segmentación o subdivisión de los puntos que conforman la superficie para extraer puntos representativos según las características locales de 
cada región. Las características 2 y 3 tienen como objetivo hacer que se interpolen correctamente los límites de la superficie (ver figura 4).

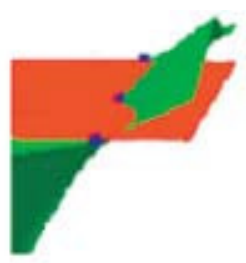

(a) Borde reproducido incorrectamente

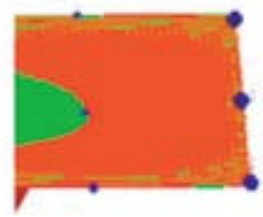

(b) Borde correctamente reproducido.

Figura 4. Efectos de la selección de centros en la reproducción del borde.

El error en la interpolación de los límites es debido a que el comportamiento de la superficie más allá de los límites de los centros de referencia está dado por la naturaleza del método utilizado. Es decir, si no se posee información en los límites de las superficies al construir el interpolante es posible que la superficie generada descienda o se eleve de forma incorrecta, por lo tanto, los bordes no se representan correctamente. En la figura 5, las flechas indican la dirección en la cual las dos superficies se extienden, esto es debido a que los puntos de referencia están alejados del borde y no es posible reproducir correctamente la superficie desde el borde hasta los centros de interpolación.

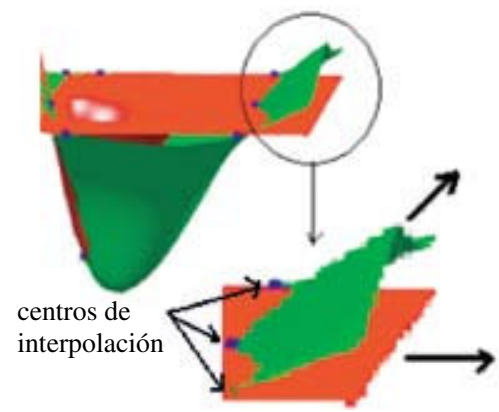

Figura 5. Interpolación incorrecta de los bordes (Roja: Superficie original, Verde: superficie interpolada).
Los puntos que cumplen con las características 2 y 3 son extraídos de los contornos que previamente se han clasificado como preservables y el conjunto de puntos que cumple la característica 1 son establecidos mediante clusterización.

\section{Selección de centros mediante clusterización}

Hay una razón teórica que indica que la interpolación es óptima si los centros están situados en los datos del conjunto inicial [22], es decir, que el conjunto de centros de interpolación sea un subconjunto de los datos a interpolar. Los experimentos con la localización de centros se han focalizado en los métodos de aproximación [18]. Aunque la función típica de minimización la constituyen los mínimos cuadrados, otras funciones objetivas también se han considerado para la minimización [14, 21]. El objetivo es obtener una buena aproximación del objeto representado por el conjunto de puntos con un subconjunto de éste. Esto reduce el tiempo de cómputo empleado en la construcción y la evaluación del interpolante que aproxime la geometría del objeto. Los resultados indican que los ahorros significativos en costo computacional pueden resultar en el costo de introducir errores muy pequeños [11], es decir, la representación digital obtenida luego de una reducción siempre será afectada por un incremento en el error.

Esta etapa implementa una técnica general de clusterización mediante crecimiento de regiones, dado que clusterizar la superficies no suele ser costoso computacionalmente si se establecen las estructuras de datos eficientemente. El algoritmo consiste en calcular previamente una estimación de la curvatura de las superficies en cada punto $P$. La estimación de la curvatura es calculada resolviendo una matriz de covarianza formada utilizando un vecindario cercano y de tamaño fijo en cada uno de los puntos así:

$$
M C=\frac{1}{n-1} \sum_{i=1}^{n}\left(p_{i}-\bar{p}\right)\left(p_{i}-\bar{p}\right)^{T}
$$

donde $n$ es el número de vecinos al punto $p$ y $\bar{p}$ se define como $\bar{p}=\frac{1}{n} \sum_{i=1}^{n} p_{i}$

La estimación de la aproximación de la curvatura en $p$ es [30]:

$$
C=\frac{\lambda_{0}}{\lambda_{0}+\lambda_{1}+\lambda_{2}}, \text { donde } \lambda_{0} \leq \lambda_{1} \leq \lambda_{2}
$$


Note que el vector propio asociado a $\lambda_{0}$ indica la dirección de la menor variación de los datos, dirección que corresponde al vector normal de la superficie en el punto $p$. De tal forma que $C$ indica cuantitativamente la variación de la superficie respecto al plano tangente, y constituye una medida de la variación de la superficie.

Una vez obtenida la aproximación de la curvatura, el siguiente paso consiste en calcular una clusterización de las superficies, de tal forma que la varianza de cada clúster no supere un umbral $\beta$ establecido. La descripción general de este procedimiento se presenta en el Algoritmo $1 \mathrm{y}$ figura 6 respectivamente.

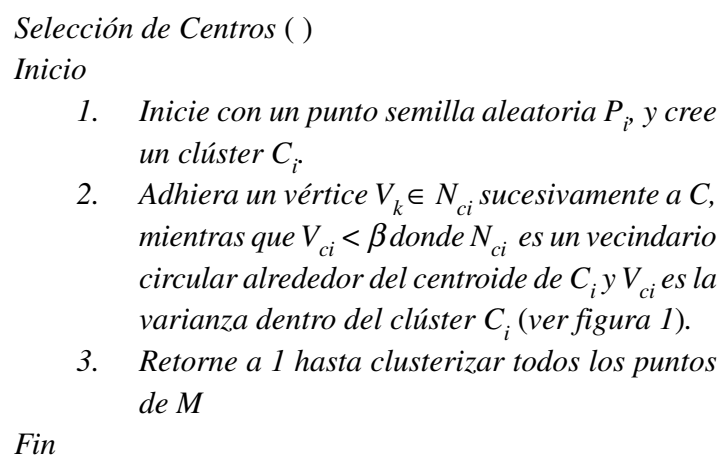

Algoritmo 2: Método propuesto para la selección de centros.

La cantidad de clúster obtenidos se comporta inversamente al valor de la varianza, a medida que la varianza aumenta, menor número de clúster son obtenidos. La varianza tendrá una incidencia directa sobre la suavidad y la calidad de la superficie interpolada, porque ésta determina la cantidad de centros con los cuales se aproximará la superficie. La técnica de clusterización tiene la propiedad de representar completamente las variaciones de la superficie, característica que no se presenta en técnicas de selección aleatoria, en la cual puede presentarse ausencia de centros en regiones pequeñas que generan altos errores y causan la interpolación iterativa del interpolante.

\section{Cálculo del interpolante}

Una vez que ha sido seleccionado el subconjunto de puntos o centros de interpolación, el siguiente paso es estimar el interpolante. El cálculo del interpolante es realizado utilizando RBF de soporte global de igual forma como se reporta en la literatura $[4,10]$.

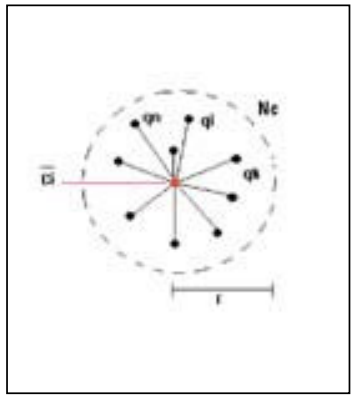

(a)

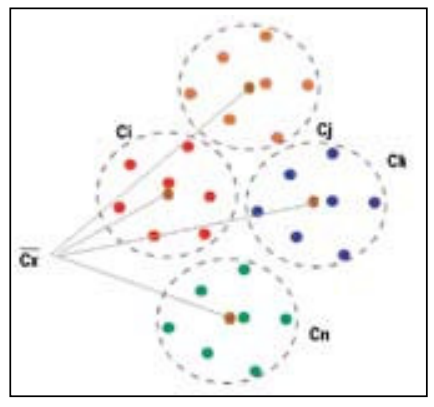

(b)
Figura 6. (a) Definición de vecindario de cada centroide, (b) Centroides de los clúster no pertenecen al conjunto de datos.

Las funciones de base radial son funciones circularmente simétricas, centradas en un punto llamado centro. Para calcular el interpolante de una RBF se considera que se tiene un conjunto de puntos $p=\left\{p_{1}, \ldots, p_{N}\right\}$ tomados desde la superficie original $S$ del objeto real. El objetivo principal es construir una función $y=f(x)$ en la cual el conjunto de ceros de la función $f(x)=0: \in x \mathfrak{R}^{3}$ que satisfacen la ecuación aproxime a la superficie $\mathrm{S}$ a partir de los datos del conjunto $\mathrm{P}$.

Típicamente la interpolación construye una función $y=f(x)$ interpolando el conjunto $\mathrm{P}$ de la siguiente manera:

$$
f(x)=\sum_{p_{i} \in p}\left[g_{i}(x)+\lambda_{i}\right] \phi_{\sigma}\left(\left\|x-C_{i}\right\|\right)
$$

Donde $\phi_{\sigma}(r)$ es la función base, el conjunto de valores $\lambda_{i}$ es el conjunto de pesos asociado a cada centro, $g_{i}$ es típicamente un polinomio de segundo o tercer grado y el conjunto $C_{\mathrm{i}}$ es denominado el conjunto de centros. Los parámetros $\phi_{\sigma}(r), C_{i}$ y $g_{i}$ son conocidos, de tal forma que $\lambda_{i}$ es estimado resolviendo el sistema de ecuaciones.

\section{Recorte de la superficie}

La obtención de una curva 3-D situada sobre una superficie se puede hacer de diversas maneras. En este trabajo se utiliza una definición de recorte como la intersección de las superficies del objeto y la superficie definida por la región encerrada por cada curva de contorno. De esta manera se pueden modelar objetos tridimensionales como intersección con curvas en el espacio. Existen algunos algoritmos que resuelven la intersección entre superficies, tanto de manera analítica como por aproximación y el recorte de la superficie dada una curva ha sido trabajado ampliamente en la literatura [28, 29]. 


\section{EXPERIMENTOS Y RESULTADOS}

\section{Determinación del umbral de torsión}

Para establecer los parámetros del algoritmo se realizaron pruebas para determinar, experimentalmente, el umbral de torsión para la clasificación de huecos mediante la medida de un conjunto de huecos generados sintéticamente sobre la superficie de una esfera (ver figura 7) y un conjunto de huecos obtenidos de objetos reales (ver figura 8). La generación sintética de huecos permite el análisis de una forma controlada de la variación del contorno. Como resultado del trabajo experimental se encontró que el valor adecuado generalmente para la correcta clasificación es 0,1 .

\section{Reconstrucción de objetos reales}

Para probar la funcionalidad del método y la efectividad de la detección y clasificación de anomalías preservables se realizaron pruebas sobre datos de rango que representan objetos con discontinuidades reales. La figura 9 muestra los resultados intermedios del proceso de reconstrucción preservando las discontinuidades sobre el objeto Máscara. Este objeto no presenta discontinuidades de anomalía, sus dos únicas discontinuidades pertenecen al modelo real. Las medidas para las estimaciones de la torsión de las discontinuidades detectadas son de 0,0568; 0,0874 respectivamente de izquierda a derecha para las discontinuidades que denotan la zona de los ojos. El borde externo es igualmente almacenado debido a que encierra el objeto completamente, este borde es preservado para el recorte final del contorno de la superficie.

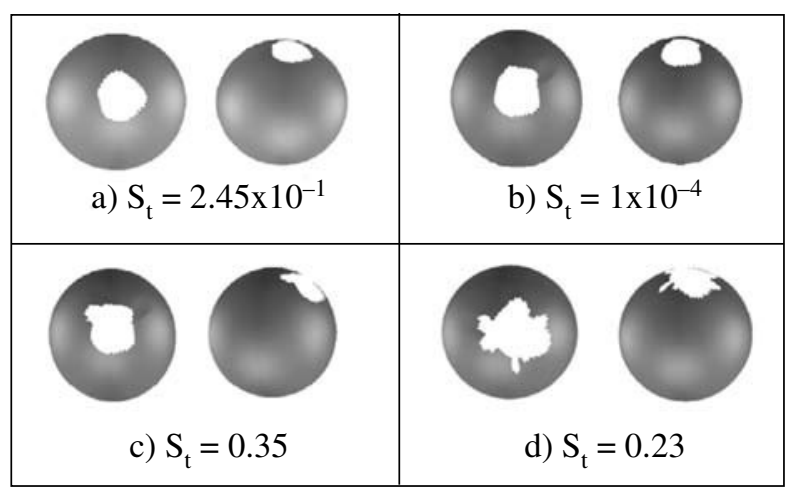

Figura 7. Torsión estimada de la curva de contorno para cuatro casos sintéticos.

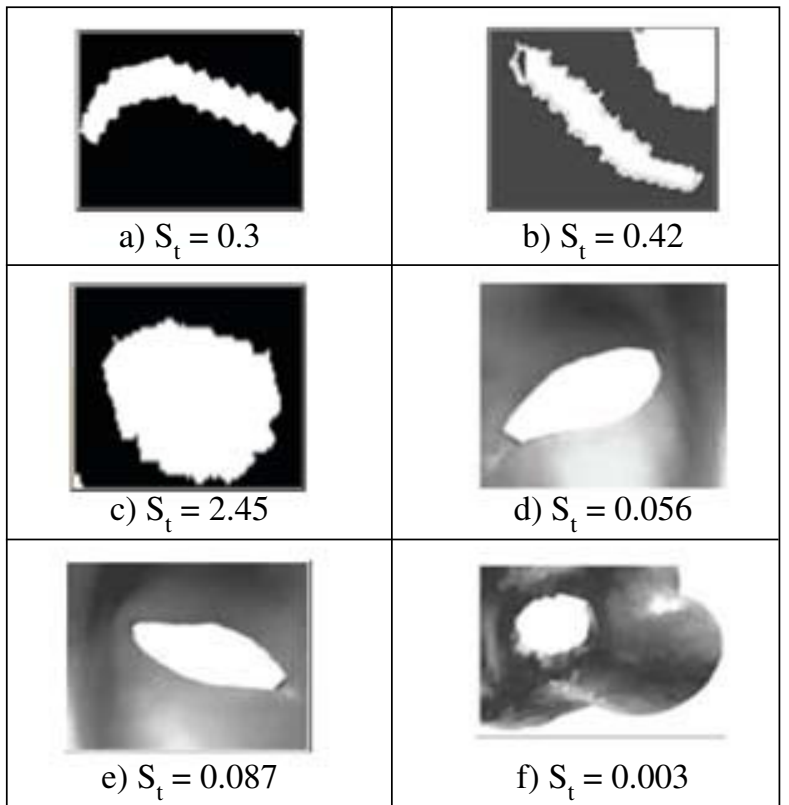

Figura 8. Torsión estimada de la curva de contorno para seis casos reales: a, b, c pertenecen anomalías en el censado del objeto, $\mathrm{d}$, e y $\mathrm{f}$ son huecos que se encuentran en el objeto real.

La figura 10 muestra los pasos intermedios del proceso de reconstrucción con preservación de discontinuidades en el objeto Stanford's Bunny con discontinuidades que pertenecen al objeto real. Las medidas para las estimaciones de la torsión de la curva de contorno de las dos discontinuidades son de 0,0046 y 0,0030 respectivamente de izquierda a derecha en la figura $11 \mathrm{~b}$. Estas discontinuidades por tener un valor estimado inferior a 0,1 se preservan y son recuperadas en la etapa final del proceso (ver figura 10e y $10 \mathrm{f}$ ).

\begin{tabular}{|l|l|}
\hline a) Imagen original & b) Contornos detectados \\
\hline & \\
\hline
\end{tabular}




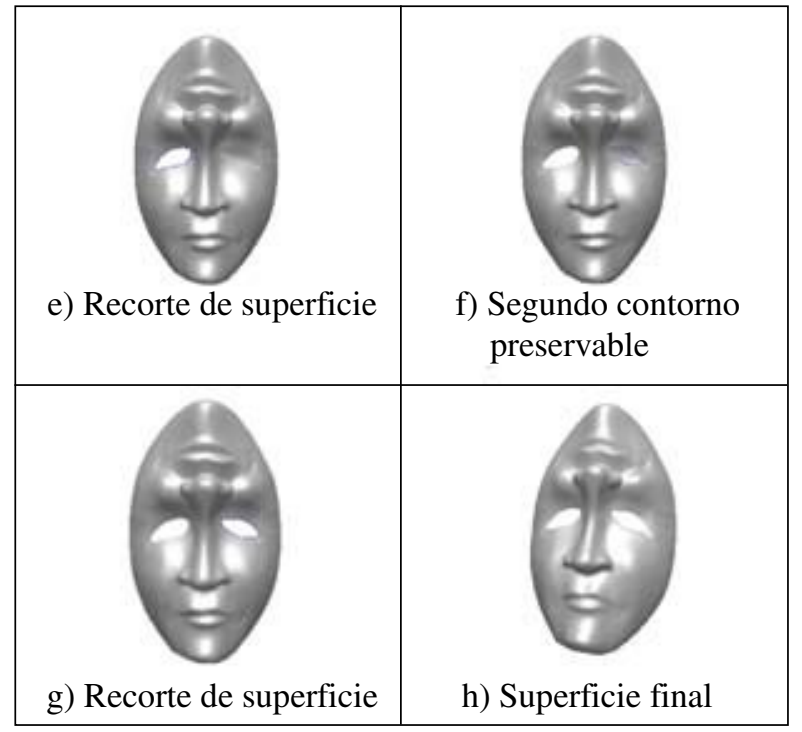

Figura 9. Preservación de discontinuidades reales en el objeto Máscara.

\section{CONCLUSIONES}

Se ha propuesto un método para el ajuste de superficies de objetos de forma libre mediante interpolación de datos dispersos utilizando funciones de base radial que preservan y corrigen discontinuidades automáticamente, mediante un método de selección de centros de interpolación y un método de identificación de discontinuidades. Este método extiende los métodos clásicos de reconstrucción al permitir representar superficies discontinuas de forma sencilla y eficiente.

La selección de centros para la generación de la interpolación es realizada mediante una clusterización basada en curvaturas de la superficie. La clusterización garantiza que cada segmento de la superficie tiene representación en el modelo interpolado [24].

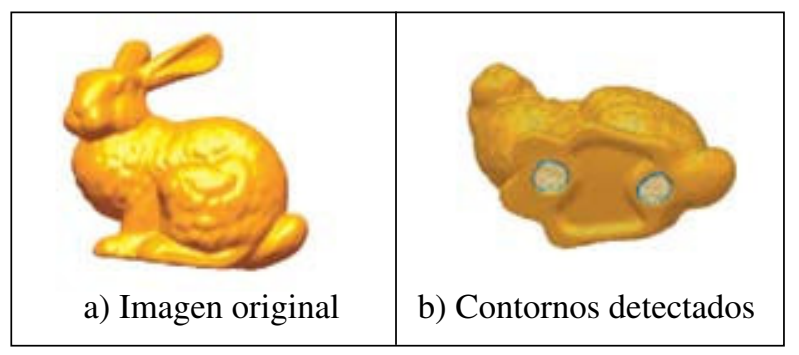

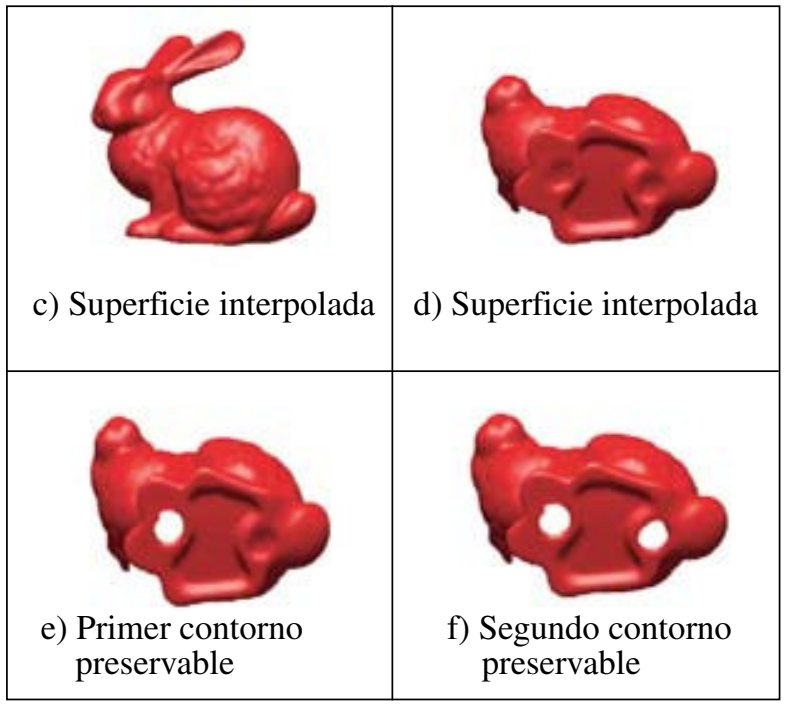

Figura 10. Preservación de discontinuidades reales en el objeto Stanford's Bunny.

La estimación de la varianza de la torsión de la curva de los contornos permite obtener una aproximación del nivel de suavidad. En general, los huecos que presentan un alto nivel de suavidad según esta estimación corresponden a aquellos huecos presentes en el objeto al momento del censado. Esto parece deberse al hecho de que el proceso de censado no incluye sobresaltos ni variaciones bruscas, que generalmente aparecen cuando el procedimiento de adquisición falla. Los resultados muestran que es posible modificar un proceso de reconstrucción global mediante el recorte de superficie en regiones en donde se presenta un contorno suave.

Adicionalmente, para la preservación de los contornos exteriores de la superficie, los vértices límites son incluidos en la matriz de interpolación, así como los vértices que describan cada discontinuidad al interior de la superficie.

La limitación principal del método consiste en el hecho de tener que formar información acerca de la conectividad de los puntos para la detección de los contornos. Sin embargo, es posible realizar una relación de vecindad directamente del conjunto de puntos basado en una estructura de datos de árbol k-d, lo que reduce la complejidad a una magnitud logarítmica. Los resultados muestran que las discontinuidades presentes en el objeto real se mantienen debido a su suavidad en el contorno; sin embargo, la estimación correcta del umbral es aún un problema, debido a que factores como la escala, la densidad de muestreo y la geometría del objeto hacen que 
el nivel adecuado de tolerancia en la variación cambie numéricamente. Finalmente, el interpolante es calculado y generado también en las zonas con falta de información, por lo que la eficiencia del método no se reduce con los tamaños de las discontinuidades presentes.

Los trabajos futuros se deberían orientar a la generación de superficies que incluyan como restricción la generación de puntos dentro de las regiones de los huecos que se deben mantener.

\section{REFERENCIAS}

J. Carr. "Reconstruction and representation of free-form objects with radial basis function". 2001.
[10] J. Carr, R. Beatson, B. McCallum, W. Fright T. McLennan and T. Mitchell. "Smooth surface reconstruction from noisy range data". GRAPHITE '03: Proceedings of the 1st international conference on Computer graphics and interactive techniques in Australasia and South East Asia, pp. 119-ff. New York, NY, USA. ACM Press. 2003.

[11] R. Carlson and B. Natarajan. "Sparse approximate multiquadric interpolation". Computers and Mathematics with Applications. Vol. 26, pp. 99-108. 1994.

[12] J. Duchon. "Spline minimizing rotation-invariant seminorms in sobolev spaces". Constructive Theory of Functions of several Variables. Vol. 571, pp. 85-100. 1997.

[13] M. Floater and A. Iske. "Multistep scattered data interpolation using compactly supported radial basis functions". Journal of Comp. Appl. Math. Vol. 73, pp. 65-78. 1996.

[14] F. Girosi. "Some extensions of radial basis functions and their applications in artificial intelligence". Computers and Mathematics with Applications. Vol. $24 \mathrm{~N}^{\mathrm{o}}$ 12, pp. 61-80. 1992.

[15] W. Grimson. "From Images to Surfaces: A Computational Study of the Human Early Visual System". MIT Press, Cambridge, MA. 1981.

[16] N. Kojekine and V. Savchenko. "Surface reconstruction based on compactly supported radial basis functions". Geometric modeling: techniques, applications, systems and tools, pp. 218-231. Kluwer Academic Publishers. 2004.

[17] D. Marr and T. Poggio. "A theory of human stereo vision”. Technical report, Cambridge, MA, USA. 1997.

[18] McMahon and R. Franke. "Knot selection for least squares thin plate splines". SIAM Journal on Scientific and Statistical Computing. Vol. 13 $\mathrm{N}^{\mathrm{o}}$ 2, pp. 484-498. 1992.

[19] Y. Ohtake. "3D scattered data approximation with adaptive compactly supported radial basis functions". In SMI '04: Proceedings of the 
Shape Modeling International 2004 (SMI'04). IEEE Computer Society, pp. 31-39. Washington, D.C., USA. 2004.

[20] M. Powell. "The theory of radial basis function approximation", in Advances in Numerical Analysis II, W.A. Light, ed., Clarendon Press, Oxford, pp. 105. 1990.

[21] K. Salkauskas. "Moving least squares interpolation with thin-plate splines and radial basis functions". Computers and Mathematics with Applications. Vol. $24 \mathrm{~N}^{\circ} 12$, pp. 177-185. 1992.

[22] R. Schaback. "Creating surfaces from scattered data using radial basis functions". In M. Daehlen, T. Lyche, and L. L. Schumaker, editors, Mathematical Methods in AGD III. Vanderbilt Press. 1995.

[23] H. Wendland. "Piecewise polynomial, positive definite and compactly supported radial functions of minimal degree". Advances in computational Mathematics 4, pp. 389-396. 1995.

[24] G. Sánchez. "Selección de centros de Interpolación de funciones de Base Radial para el Ajuste de superficies de Objetos de forma libre". CLEI-XXXII Conferencia Latinoamericana de Informática. Santiago de Chile, Chile. Agosto de 2006.

[25] N. Amenta, M. Bern and M. Kamvysselis. "A new voronoi-based surface reconstruction algorithm". Proceedings of the SIGGRAPH'98, pp. 415-421. 1998.

[26] M. Gopi and S. Krishnan. "A fast and efficient projection based approach for surface reconstruction”. International Journal of High
Performance Computer Graphics, Multimedia and Visualisation. Vol. $1 \mathrm{~N}^{\mathrm{o}}$ 1, pp. 1-12. 2000.

H. Hoppe, T. De Rose, T. Duchamp, J.A. McDonald and W. Stuetzle. "Surface reconstruction from unorganized points". Proceedings of the IGGRAPH'92, pp. 71-78. 1992.

[28] G. Farin. "Curves And Surfaces For Computer Aided Geometric Design". A Practical Guide. Academic Press, Inc. ISBN 0-12-249052-5. 1993.

I.D. Faux and M.J. Pratt. "Computational Geometry for Design and Manufacture". Ellis Horwood Ltd, Publishers. ISBN 0-85312-1141. 1979.

[30] M. Pauly, M. Gross and L. Kobbelt. "Efficient simplification of point-sampled surfaces". In: Proceedings of IEEE visualization 2002, pp. 163-170. 2002.

[31] J. Branch, F. Prieto and P. Boulanger. "Automatic Hole-Filling of Triangular Meshes Using Local Radial Basis Function". In Proceedings of the Third international Symposium on 3D Data Processing, Visualization, and Transmission (3dpvt'06). 3DPVT. IEEE Computer Society, Washington, DC, 727-734. June 14-16, 2006.

[32] Brian Curless and Marc Levoy. "A volumetric method for building complex models from range images”. In SIGGRAPH ‘96: Proceedings of the 23rd annual conference on Computer graphics and interactive techniques, pp. 303312, New York, NY, USA. ACM Press. 1996. 